Was ist neu in der medikamen-
tösen Therapie?
Wir halten Sie auf dem
Laufenden mit Berichten von
Kongressen und Symposien der
pharmazeutischen Industrie.

\title{
Zielorgan Niere
}

\section{Neues Antidiabetikum hilft dem Körper, Glukose loszuwerden}

\section{Derzeit befindet sich ein neues Wirkprinzip zur Blutzuckersenkung in der klinischen Prüfung: SGLT2- Hemmer fördern die Glukoseaus- scheidung mit dem Urin.}

- SGLT2-Hemmer blockieren den Natrium-Glukose-Transporter 2 (SGLT2) in den Nierentubuli. Dieser Transporter ist für $90 \%$ der Glukoserückresorption in der Niere verantwortlich. Wird er gehemmt, gelangt weniger Glukose aus der Niere zurück ins Blut, die überschüssige Glukose wird mit dem Harn ausgeschieden. Aufgrund des insulinunabhängigen Wirkmechanismus könnten SGLT2-Hemmer im Prinzip in allen Stadien des Typ-2-Diabetes eingesetzt werden, erklärte Prof. Juris Meier, Bochum.

\section{Gute blutzuckersenkende Wirkung}

Der am weitesten in der klinischen Prüfung fortgeschrittene SGLT2-Hemmer ist Dapagliflozin. In einem großen Phase-III-Studienprogramm wurde er in Monotherapie und in Kombination mit anderen Antidiabetika geprüft. „Die $\mathrm{HbA}_{1 c}$-Reduktion von Dapagliflozin ist vergleichbar mit der anderer oraler Antidiabetika", erklärte Meier.

In Monotherapie führte der Wirkstoff bei Typ-2-Diabetikern mit einem Ausgangswert von 7,8-8,0\% innerhalb von 24 Wochen zu einer Reduktion des $\mathrm{HbA}_{1 \mathrm{c}}$ um etwa 0,6-0,9 Prozentpunkte (Placebo: $-0,23 \%$; Ferrannini E et al. Diabetes Care 2010; 33: 2217-2224). In Kombination mit Metformin waren unter dem SGLT2-Hemmer bessere Langzeiterfolge hinsichtlich des $\mathrm{HbA}_{1 c}$-Werts zu beobachten als unter dem Sulfonylharnstoff Glipizid, wie eine zwei Jahre dauernde Studie ergeben hat (Nauck M et al. Poster beim ADA-Kongress, Juni 2011). Interessant könnte auch die Kombination mit Insulin sein. Hier verbesserte der SGLT2-Hemmer den $\mathrm{HbA}_{1 c}$-Wert innerhalb von zwölf Wochen um etwa 0,7 Prozentpunkte im Vergleich zu Placebo (Wilding JPH et al. Diabetes Care 2009; 32: 1656-1662).

Positive Nebeneffekte: In allen Studien sank unter dem SGLT2-Hemmer das Gewicht um etwa 3-4 kg sowie der Blutdruck um etwa 3-4 mmHg. Ein gesteigertes Hypoglykämierisiko war nicht zu beobachten.

\section{Mögliches Krebsrisiko}

Die unerwünschten Nebenwirkungen des neuen Wirkprinzips könnten allerdings problematisch sein. Einige Tumoren waren unter Dapagliflozin häufiger aufgetreten als in den Vergleichsgruppen, v.a. Blasen-, Brust- und Prostatakarzinome. Das mögliche Krebsrisiko veranlasste die FDA dazu, weitere Studiendaten zu fordern.

Hinzu kommt, dass die Häufigkeit von Genitalinfektionen bei Frauen signifikant angestieg. Das absolute Risiko sei aber überschaubar, erklärte Meier, es liege bei etwa 7\%. Außerdem litten die Patienten vermehrt unter Harnwegsinfektionen.

\footnotetext{
- Dr. Judith Neumaier

Quelle: Satellitensymposium „Insulinabhängige und -unabhängige Therapien des Typ-2 Diabetes - Was bringt die Zukunft", Kongress "Diabetologie grenzenlos", München, Februar 2012 (Veranstalter: AstraZeneca und BristolMyers Squibb)
}

\section{Kurz notiert}

Was Sie in der aktuellen „Schmerzmedizin“ lesen $>$ Die erste Ausgabe der Zeitschrift "Schmerzmedizin" (bisher "Schmerztherapie"), das offizielle Organ der Deutschen Gesellschaft für Schmerztherapie, respektive der deutschen Gesellschaft für Schmerzmedizin (DGS), gibt eine Vorschau auf den Deutschen Schmerzund Palliativtag 2012 in Frankfurt/ Main. Im Editorial erläutert Dr. Gerhard H. H. Müller-Schwefe, Göppingen, warum die Gesellschaft wie auch die Zeitschrift ihren Namen geändert haben. Weitere Themen: multimodale Therapie bei Patienten mit chronischen Rückenschmerzen, DGS-Pilotprojekt Kompetenzapotheke Schmerz, Schmerzoffensive der DGS. Bezug über: Deutsche Gesellschaft für Schmerztherapie e.V., Frau Nitsche, Adenauer Allee 18, D-61440 Oberursel.

StK

SilverStar Förderpreis 2012 - Auch in diesem Jahr wird der mit 25000 Euro dotierte SilverStar Förderpreis verliehen für Projekte oder Initiativen, die zu einer besseren Versorgung älterer Diabetiker beitragen. Bis zum 30. Juni 2012 können sich Gesundheitsprofis (z. B. Ärzte, Pflegekräfte oder Apotheker), Angehörige, Freunde, Nachbarn oder Betroffene bewerben. Weitere Infos unter: www. silverstar-preis.de. Berlin-Chemie 